\title{
Analysis of stripline right-angle bend with slantwise corner cut based on Foster-type equivalent network and exact calculation of eigenmode
}

\author{
Takaharu Hiraoka Hsu, Jui-Pang \\ Faculty of Engineering, KANAGAWA University \\ 3-27-1 Rokkakubashi Kanagawa-ku Yokohama 221-8686 Japan \\ E-mail : hiraoka@kyolab.ee.kanagawa-u.ac.jp
}

Stripline right-angle bend with slantwise corner cut is an important component for microwave circuit. Especially, analytical determination of the exact optimum cut is important for computer aided design of microwave circuit. Equivalent network for this structure is given by Foster-type network based on mode theory and can be used for analysis of practical bend characteristics. The key step for this analysis is how exactly to calculate eigenmode of the planar junction up to necessary number. Therefore, we propose a new method using three stage cascade-connected stripline configuration and magnetic wall symmetry. In this paper, we explain the above method in detail and calculate the eigenmode for various cut parameters. Finally, frequency characteristics of the right-angle bend with slantwise corner cut are calculated for various cut parameters, resulted in the determination of the optimum cut.

\section{Introduction}

Stripline right-angle bend with slantwise corner cut shown in Fig. 1(a) is an important component for microwave circuit ${ }^{[1]}$. Especially, analytical determination of the exact optimum cut is important for computer aided design of microwave circuit. Equivalent network for this 2-dimensional structure is given by Foster type network as shown in Fig. 1(b) based on mode theory ${ }^{[2],[3]}$ and can be used for analysis of practical bend characteristics. The key step for this analysis is how exactly to calculate eigenmode of the planar junction $S$ in Fig.1(a) up to necessary number. Therefore, we propose a new method using three stage cascade-connected stripline configuration as shown in Fig.3(b) and magnetic wall symmetry. In this paper, we explain the above method in detail and calculate the eigenmode for various cut parameters $(=c / a$ in Fig.2). Finally, frequency characteristics of the right-angle bend with slantwise corner cut are calculated for various cut parameters, and the optimum cut is discussed and roughly determined.

\section{Equivalent network based on mode theory}

We assume the structure can be modelled by $2 \mathrm{D}$ planar circuit with magnetic side wall. Then, right-angle bend structure is divided into waveguide and junction, whose equivalent network is given by Foster-type network as shown in Fig.1 $(b)$ or vector representation in Fig.1(c) consisting of multi-transmission line for waveguide, infinite number of resonators for planar junction and

Table 1 Eigenmode system for waveguide and the corresponding network parameters

$$
\begin{aligned}
& c_{p}^{i}\left(s^{i}\right)=\sqrt{\varepsilon_{p}} \cos \frac{p \pi}{W^{i}} s^{i} \quad \frac{1}{W^{i}} \int_{0}^{W^{i}} c_{p}^{i}\left(s^{i}\right) c_{p^{\prime}}^{i}\left(s^{i}\right) d s^{i}=\delta_{p p^{\prime}} \\
& \gamma_{p}^{i}=\sqrt{\left(\frac{p \pi}{W^{i}}\right)-k^{2}} \quad Z_{C_{p}}^{i}=\frac{j \omega \mu d}{\gamma_{p}^{i} W^{i}}
\end{aligned}
$$

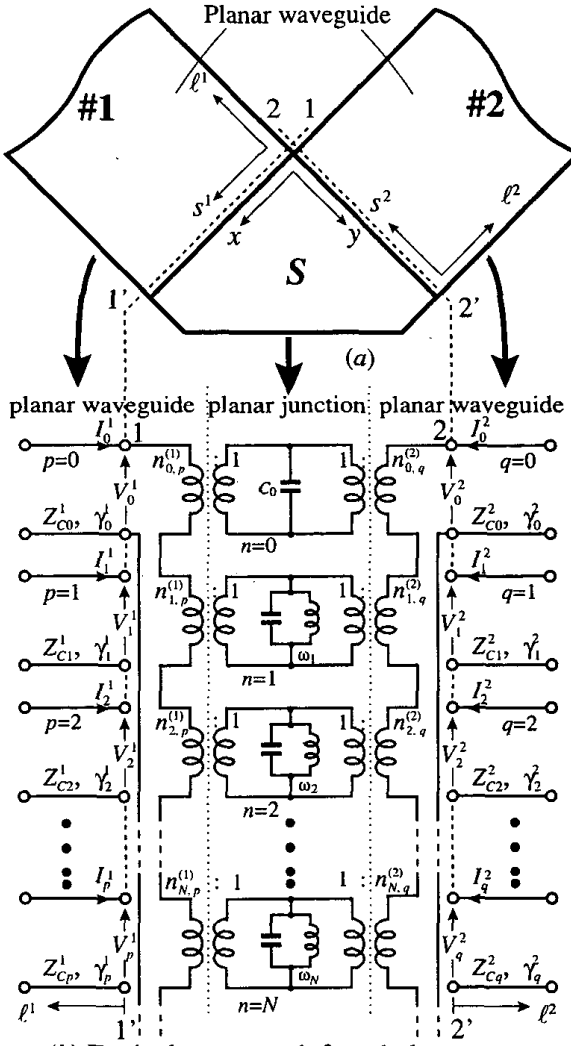

(b) Equivalent network for whole structure

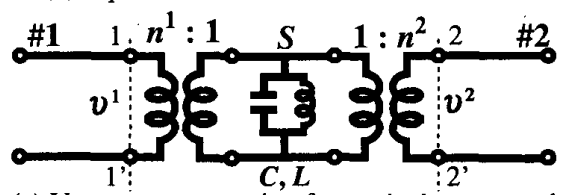

(c) Vector representation for equivalent network

Fig.1 Right-angle bend with slantwise corner cut and its equivalent network representation 
ideal transformer for coupling between waveguide mode and resonator mode $n_{n, q}^{j}$. Eigenmode for waveguide and its network parameters are summarized in Table 1 and eigenmode for planar junction in Table 2 . From these tables, network parameters of $n$-th mode resonator and ideal transformer ratio between $n$-th mode resonator in the planar junction and $q$-th mode in the $j$ th waveguide are given by eqs.(1).

$$
\begin{gathered}
C_{0}=\varepsilon \frac{S}{d}[\mathrm{~F}] \quad L_{n}=\frac{\varepsilon \mu}{k_{n}^{2}} \frac{1}{C_{0}}[\mathrm{H}] \quad \omega_{n}=\frac{k_{n}}{\sqrt{\varepsilon \mu}} \\
n_{n q}^{j}=\frac{1}{W_{j}} \int_{0}^{W_{j}} \phi_{n}(x, y) f_{q}^{j}\left(s^{j}\right) d s^{j}
\end{gathered}
$$

\section{Calculation of eigenmode in planar junction}

In order to calculate 2D eigenmode for planar junction shown in Fig.2 (where two cases A and B are considered), we propose following new method. When two identical planar junctions of square shape with slantwise corner cut are connected back to back as shown in Fig.3(a) ( $X-X^{\prime}$ for case A, $Y-Y^{\prime}$ for case B), then whole structure can be understood as three stage cascade connected stripline as in Fig.3(b), whose equivalent network is exactly given by alternative connection of multi-transmission line and multi-port ideal transformer as shown in Fig.4. When input mode admittance matrix $\bar{Y}_{i n}^{(3,2)}$ at port 2 of waveguide $\# 3$ is given by this equivalent network, mode current column matrix at port $(3,2)$ is given by mode voltage column matrix at the same port by eq.(2)

$$
\bar{i}^{(3,2)}=\bar{Y}_{i n}^{(3,2)} v^{(3,2)}=0
$$

and must be zero because of magnetic wall(=open circuit) at port $(3,2)$, which constitutes the eigenvalue problem. Then, eigenvalue can be calculated by eq.(3).

$$
\operatorname{det}\left[\bar{Y}_{i n}^{(3,2)}\right]=0
$$

The mode voltage column matrix at port $(3,2) v^{(3,2)}$ can be calculated as an eigenvector in eq.(2) with one arbitrary constant. Then mode voltage and mode current column matrix at each port can be calculated by the equivalent network in Fig.4, which, in turn, gives field distribution of the corresponding mode. Using the normalization defined in Table 2, one arbitrary constant mentioned above is uniquely determined. Thus, eigenmodes up to necessary higher mode are calculated.

Essential problem in this method is how many width mode at each waveguide must be taken into consideration. As a typical example, convergence behavior of eigenvalue with width mode of waveguide 1 in Fig.4 is calculated in Fig.5 for $c / a=0.4$ and 8 th $\operatorname{mode}(n=8)$, which demonstrates fast convergence with width-mode

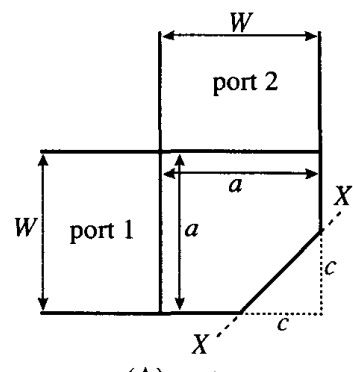

(A) $c<a$

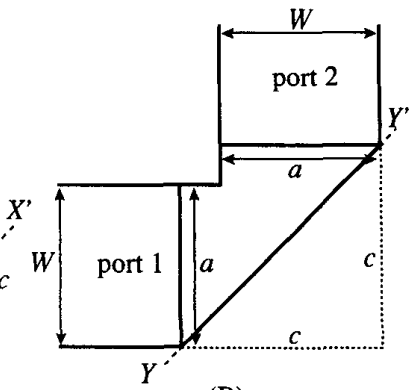

(B) $c>a$
Fig. 2 Two cases of stripline right-angle bend

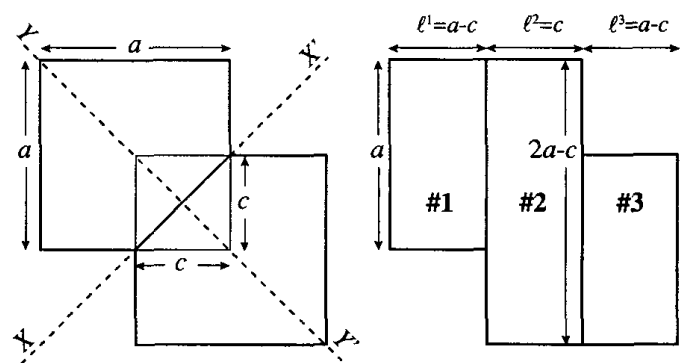

(a) Back to back connection (b) 3-stage connection of stripline

Fig.3 New method for eigenmode calculation

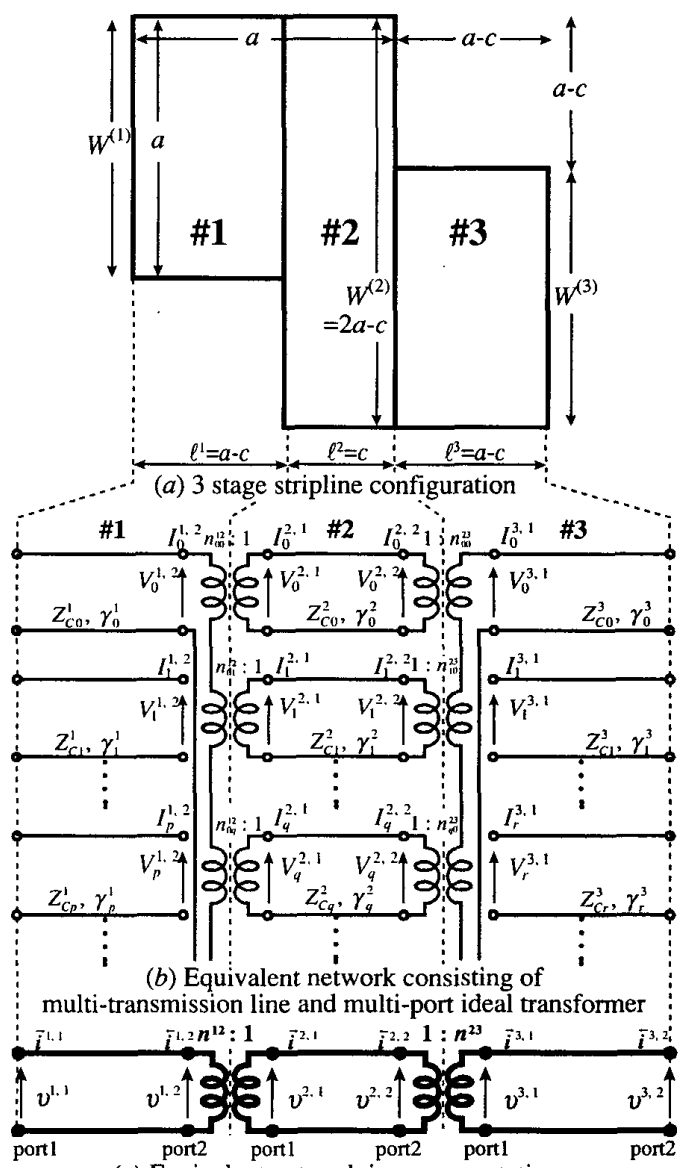

(c) Equivalent network in vector notation

Fig.4 Equivalent network of 3 stage configuration 
and 10 width modes for sufficient convergence. Therefore, through our calculation 30 width modes are taken into consideration for sure convergence. Eigenvalue for square shape with slantwise corner cut is calculated up to $k a=20$ for various cut parameter and shown in Fig. 6 as a function of cut parameter. How to derive the corresponding field distribution of eigenmode is explained Fig.7. It is interesting that 3-stage eigenmodes with magnetic wall symmetry about $X-X^{\prime}$ and $Y-Y^{\prime}$ gives eigenmode for $c_{0} / a<1$ (case A) and $c / a=2-c_{0} / a>1$ (case B) at the same time as shown by case $(d)$ in Fig.7.

\section{Calculation of the frequency characteristics}

Based on the above mode calculation, network parameters in Fig.1(b) are calculated from eqs.(1). Then, the frequency characteristics of the corner cutted right-angle bend for $W=5.0[\mathrm{~mm}], \varepsilon_{s}=2.62$ are calculated, based on the network shown in Fig.1. The essential problem in these calculations is how many modes in the waveguide and planar junction must be taken into consideration. It is already estimated ${ }^{[3]}$ that up to 5 th waveguide modes and up to eigenvalue $k a=20$ planar junction modes are enough. Final calculated results of the frequency characteristics for various cut parameters are shown in Fig.7(c). From this figure and Fig.8(a) (=detail about cut parameter of 0.8 0.9) it turns out that the optimum cut parameter is about 0.86 . Convergence behavior of transmission with eigenmode in planar junction at $6.0 \mathrm{GHz}$ is shown in Fig. $8(b)$, where abscissa corresponds eigenvalue $k a$ and all eigenmodes up to this value are taken into consideration ( 53 eigenmodes for $k a=30$ ). From this figure accuracy in Fig.8(c), where modes up to $k a=20$ are considered, is estimated to be less than $10^{-3} \mathrm{~dB}$.

\section{Discussion and conclusion}

By new mode calculation and Foster-type equivalent network, wide-band frequency characteristics of rightangle bend with slantwise corner cut are calculated. Relative error of network parameter is estimated to be $10^{-}$ ${ }^{3} \sim 10^{-4}$ for new method. However, accuracy of network parameters does not affect final character so much because of wideband frequency characteristics of the structure itself.

\section{Reference}

[1] Reinmut K. Hoffmann, "Handbook of microwave integrated circuit" pp267-309 Artech House (1987)

[2] Hsu, Jui-Pang "Analysis method for electromagnetic wave problems" edited by E.Yamashita chap.6, pp226-251 Artech house [3] Hsu, Jui-Pang, Takaharu Hiraoka and Yasushi Tabei "Analysis of stripline right-angle bend with slantwise corner cut based on eigenmode expansion method and Foster-type equivalent network" 1999 MTT-S Digest WEF2-2 pp1315-1318

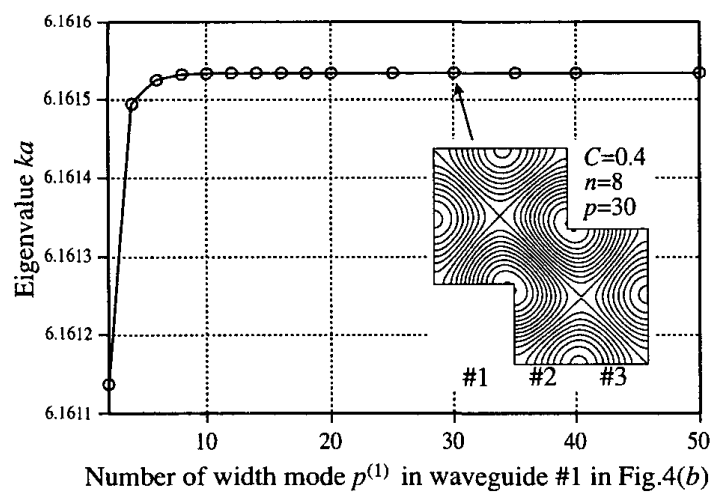

Fig.5 Convergence of 8th eigenvalue in 3-stage configuration with width mode in waveguide 1

Table 2 Eigenmode system for planar junction $\frac{\partial \varphi_{n}}{\partial x^{2}}+\frac{\partial \varphi_{n}}{\partial y^{2}}+k_{n}^{2} \varphi_{n}=0 \quad$ in $S$

$\boldsymbol{n} \cdot \operatorname{grad} \varphi_{n}=0 \quad$ on $C \quad$ (entire circumference of $S$ ) $k_{0}=0, k_{1} \leq k_{2} \leq \cdots \quad n=0,1,2, \cdots$

$\frac{1}{S} \iint_{S} \varphi_{n} \cdot \varphi_{m} d x d y=\delta_{n m} \quad$ (orthonormal) where $S$ is the area of the planar junction

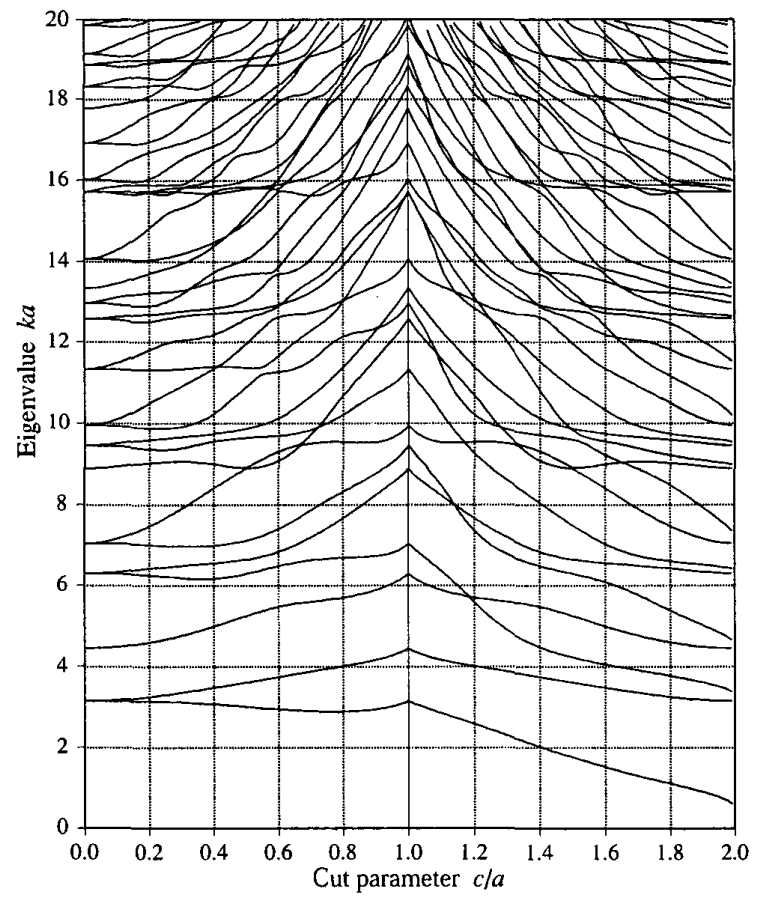

Fig.6 Eigenvalue $k a$ of square shape with slantwise corner-cut for cut parameter range $0.0 \sim 2.0$ 

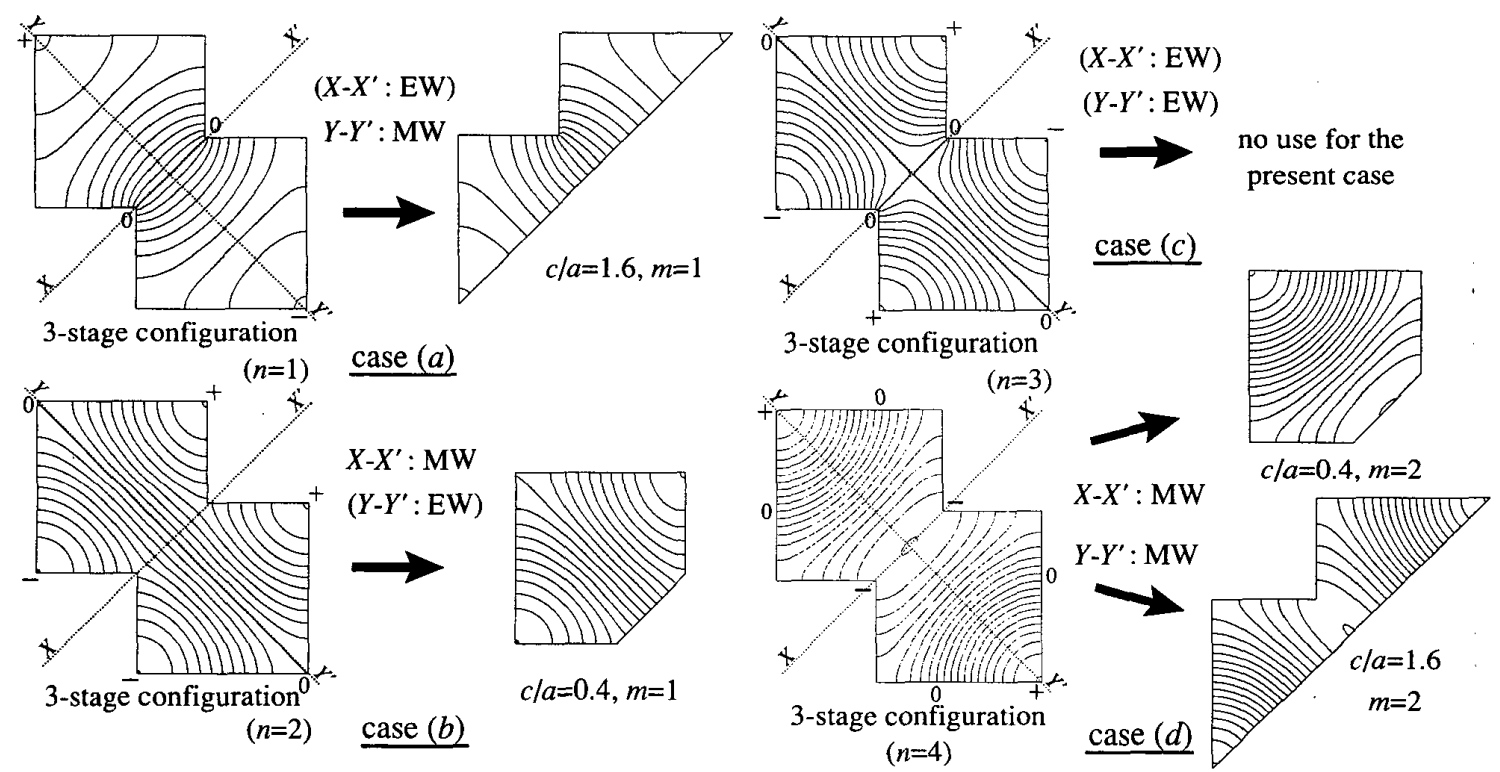

Fig.7 Derivation of mode using magnetic/electric wall symmetry about $X-X^{\prime}$ and $Y-Y^{\prime}$ for 3-stage configuration (cut parameter $c / a=0.4$ )

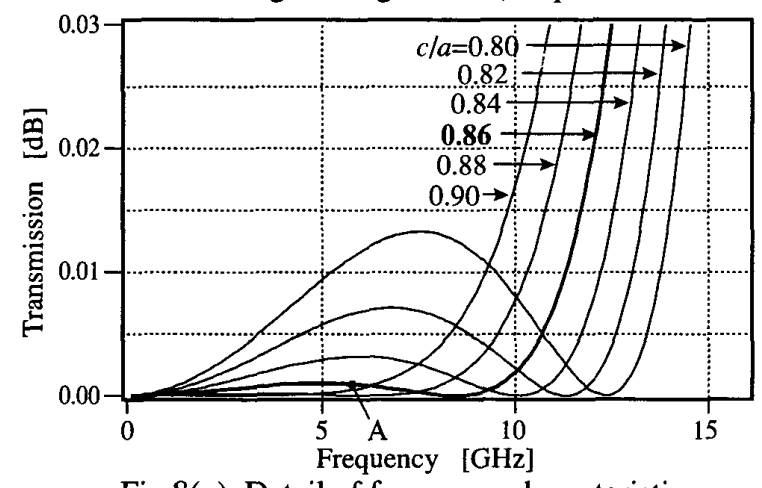

Fig.8(a) Detail of frequency characteristics $(c / a=0.8 \sim 0.9)$

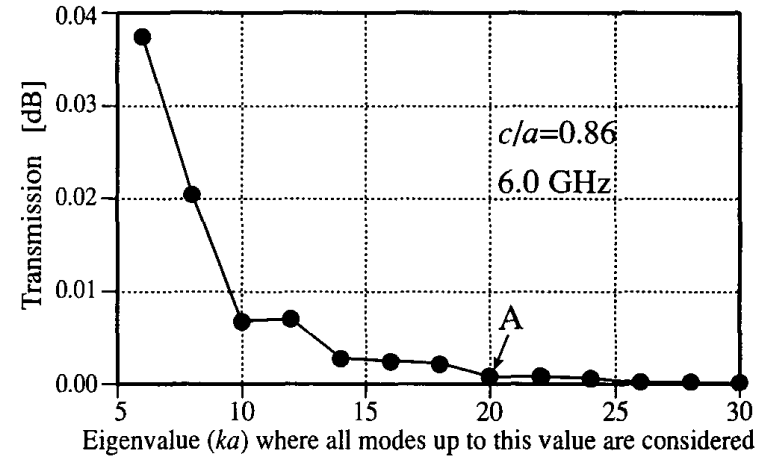

Fig. $8(b)$ Convergence behavior of transmission with eigenmode in planar junction at $6.0 \mathrm{GHz}$

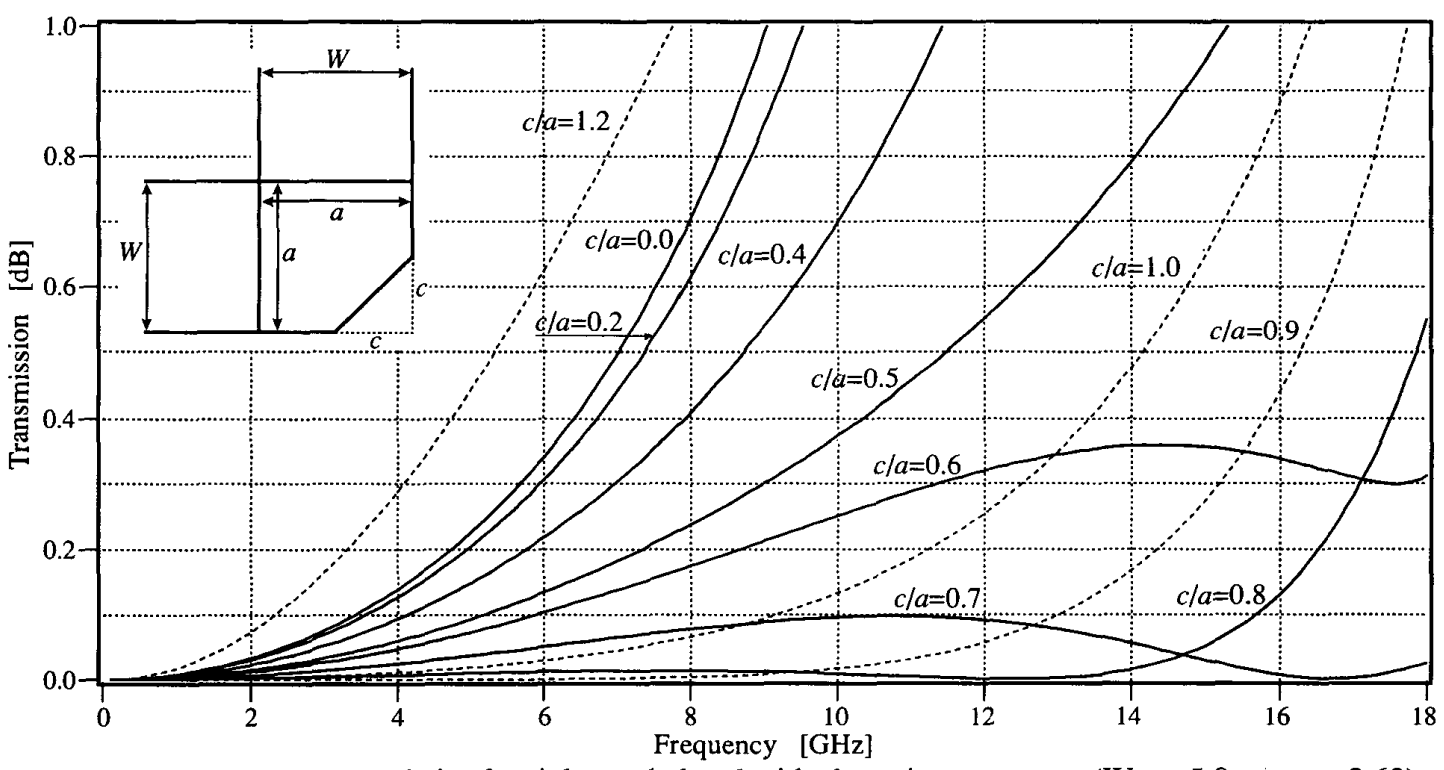

Fig.8(c) Frequency characteristics for right-angle bend with slantwise corner cut ( $\mathrm{W}=a=5.0 \mathrm{~mm}, \varepsilon_{s}=2.62$ ) 